\title{
TOURNIQUETLESS TOTAL KNEE ARTHROPLASTY WITH MODERN \\ PERIOPERATIVE PROTOCOLS DECREASES PAIN AND OPIOID CONSUMPTION IN FEMALES
}

\author{
Michael M. Kheir MD ${ }^{1}$ \\ Mary Ziemba-Davis BA ${ }^{2}$ \\ Julian E. Dilley BS ${ }^{3}$ \\ Mark Hood Jr MD ${ }^{1}$ \\ R. Michael Meneghini MD ${ }^{1,2}$ \\ ${ }^{1}$ Indiana University School of Medicine, Department of Orthopaedic Surgery, \\ Indianapolis, IN 46202 \\ ${ }^{2}$ Indiana University Health Physicians Orthopedics and Sports Medicine, \\ IU Health Saxony Hospital, Fishers, IN 46037 \\ ${ }^{3}$ Indiana University School of Medicine, Indianapolis, IN 46202
}

Corresponding Author:

R. Michael Meneghini, MD

Indiana University Health Physicians Orthopedics and Sports Medicine

Indiana University School of Medicine, Department of Orthopaedic Surgery

13100 East $136^{\text {th }}$ Street, Suite 2000

Fishers, IN 46037

Phone: 317-688-5980

Email: rmeneghi@iuhealth.org

This is the author's manuscript of the article published in final edited form as:

Kheir, M. M., Ziemba-Davis, M., Dilley, J. E., Hood, M., \& Meneghini, R. M. (2018). Tourniquetless Total Knee Arthroplasty With Modern Perioperative Protocols Decreases Pain And Opioid Consumption In Females. The Journal of Arthroplasty. https://doi.org/10.1016/j.arth.2018.06.038 


\section{TOURNIQUETLESS TOTAL KNEE ARTHROPLASTY WITH MODERN \\ PERIOPERATIVE PROTOCOLS DECREASES PAIN AND OPIOID CONSUMPTION IN FEMALES}

\section{Abstract}

Introduction: This study examined whether a modern TKA protocol without a tourniquet results in less patient-reported pain and in-hospital opioid consumption compared to TKA with a tourniquet.

Methods: A retrospective study of 203 primary unilateral cemented TKAs consecutively performed with or without tourniquet was performed. Identical perioperative pain and blood loss protocols were used in all cases. In tourniquetless TKAs, the tourniquet was not inflated at any time and sterile $\mathrm{CO}_{2}$ gas compression maximized cement interdigitation.

Results: After exclusions for scientific confounds, 184 TKAs (93 with tourniquet; 91 tourniquetless) were analyzed. Controlling for multiple covariates, females with a tourniquet reported significantly more pain $(p=0.002)$ and opioid consumption $(p<0.001)$ the first 24 hours following surgery compared to females without a tourniquet. There were no differences in pain $(p=0.192)$ or amount of opioids consumed $(p=0.203)$ among males with and without a tourniquet. Tourniquet use resulted in a significant reduction in blood loss for both females $(p \leq$ $0.040)$ and males $(p \leq 0.020)$, although the total blood savings of approximately 200 milliliters is of unknown clinical significance.

Conclusion: Avoiding tourniquet use during TKA for females may be a relatively risk-free adjunct to minimize opioid consumption during hospitalization. Further study is warranted to elucidate the factors accounting for different outcomes in females and males.

Keywords: total knee arthroplasty, tourniquet, tourniquetless, pain, opioids, blood loss 


\section{Introduction}

Pneumatic tourniquets are commonly used in total knee arthroplasty (TKA) to reduce intraoperative blood loss, enhance operative visualization, provide a cleaner field for cement penetration and fixation, and increase operative efficiency. The use of tourniquets during TKA has been a subject of debate in the scientific literature due to risks and benefits associated with their use. Systematic reviews and meta-analyses of randomized controlled trials (RCTs) show high similarity in results. Pooled analyses of 634 knees, [1] 493 patients, [2] 859 patients, [3] and 689 knees [4] all showed significantly less intraoperative blood loss in tourniquet groups, although two of these studies showed no difference in calculated ("true" or "actual") blood loss. $[1,4]$ Three of these reviews reported no statistically significant differences in the incidence of deep vein thrombosis and/or pulmonary embolism, [1-3] although two reviews [1,4] showed a greater likelihood of thrombotic events in general in tourniquet groups. Wound problems, [1] minor complications, [2] and surgical site infection [3] were all more likely when a tourniquet was used. Two systematic reviews and meta-analyses of RCTs comparing tourniquet release before and after wound closure comprised of 670 knees [5] and 1170 patients [6] reported that complications were lower when tourniquets were released prior to wound closure. Further, several studies have demonstrated that tourniquets result in greater pain in the immediate postoperative period after TKA.[7-12] Some studies additionally have noted either an increase $[7,12]$ or no difference in [11] analgesia consumption in relation to this increased pain.

In recent years, misuse and abuse of narcotic prescriptions has risen to the forefront as an urgent and grave concern. In 2011, the Executive Office of the President of the United States identified prescription drug abuse as the nations' fastest growing drug problem and issued a strong call for action. [13] Surgical specialties including orthopedics, the fifth highest opioid 
prescribing specialty group in the U.S. in 2012, prescribed $9.8 \%$ of all U.S. opioid prescriptions in that year.[14] In an evaluation of half of all prescriptions issued nationwide in 2009, orthopedic surgeons prescribed 7.7\% (6.1 million) of all opioid prescriptions, fourth behind primary care and internal medicine physicians and dentists.[15] In step with many other professional organizations, the American Academy of Orthopaedic Surgeons addressed the nations' call to action by issuing a statement on "opioid use, misuse, and abuse in orthopedics," providing recommendations for addressing excessive and inappropriate opioid consumption in orthopedic patients. [16] Subsequently, several studies have identified preoperative opioid use, age, and sex, among other factors, as strong predictors of continued opioid use after TKA. [1719] In this study, we examined the effect of tourniquet use on pain and opioid consumption in the early postoperative period following TKA performed with modern perioperative pain protocols. Blood loss in tourniquet and non-tourniquet TKAs is presented as a secondary outcome.

\section{Methods}

Study Sample

A retrospective study of 203 primary unilateral cemented TKAs consecutively performed at a single academic institution between January 2016 and March 2017 was conducted with Institutional Review Board approval. Inclusion criteria included unilateral cemented TKA secondary to primary osteoarthritis, traumatic osteoarthritis, or inflammatory arthritis. To maintain scientific validity of the study by minimizing confounding variables, patients who took antiplatelet medications except aspirin $(n=8)$, had a clotting disorder $(n=6)$, unplanned tourniquet disruption $(n=4)$, or preexisting periarticular hardware $(n=1)$ were excluded. The final analysis sample consisted of 184 TKAs, 93 of which included the use of a tourniquet and 91 
of which did not. The consecutive series of surgeries performed with a tourniquet was immediately followed by the consecutive series of cases performed without a tourniquet. To maximize the effect of limb ischemia time on the outcome variables, the tourniquetless knee group did not have a tourniquet inflated at any time including during cementation of components. Carbon dioxide compression gas (CarboJet ${ }^{\circledR} \mathrm{CO}_{2}$ Bone Preparation System, Kinamed Incorporated, Camarillo, CA) was used in tourniquetless knees to optimize cement penetration.

\section{Surgical Procedure}

Surgeries were performed by a single fellowship-trained arthroplasty surgeon. A median parapatellar approach was used for all procedures. Standard coronal plane femoral bone cuts were made with computer-aided navigation (Stryker Navigation, Kalamazoo, MI) and tibial cuts were performed with an extramedullary cutting guide. The same cruciate retaining knee implant was used in all cases (EMPOWR 3D Knee ${ }^{\mathrm{TM}}$, DJO Surgical, Vista, CA). Surgeries were performed with standardized light general anesthesia, low-dose intrathecal/single-shot spinal injection of $25 \mathrm{mcg}$ fentanyl and $4.5 \mathrm{mg}$ bupivacaine, and a periarticualar injection of $0.2 \%$ (200mg) ropivacaine, $0.5 \mathrm{mg}$ epinephrine, $80 \mathrm{mcg}$ clonidine and $30 \mathrm{mcg}$ ketorolac to equal 101.3 $\mathrm{mL}$ total volume immediately following component fixation. Dosing was identical in all patients, except that ketorolac was removed for patients with renal insufficiency. Multimodal perioperative pain protocols were used in all cases and consisted of preoperative oxycodone, Lyrica, Celebrex (or ketorolac if sulfa allergic) and oral Tylenol 24 hours prior to surgery. Postoperative protocols were identical with the addition of oxycontin if under 70 years of age, and tramadol if 70 or older. The same modern perioperative pain control, clinical, and rehabilitation protocols were used for all patients. 
Prior to closure of the arthrotomy, a medium hemovac drain was placed in all knees and one gram of topical tranexamic acid was applied to the site. When tourniquets were used, the tourniquet was inflated to a pressure of $250 \mathrm{~mm} \mathrm{Hg}$ from surgical incision until the postoperative sterile dressing was applied. A pad was applied between the skin and the tourniquet cuff to protect the skin.

\section{Measurements}

Patient sex, age in years, body mass index (BMI), American Society of Anesthesiologists Physical Status (ASA-PS) classification, procedure time in minutes, tourniquet use (yes/no), tourniquet (limb ischemia) time in minutes, hospital length of stay (LOS) in days, and preoperative presence of lumbar spine disease, fibromyalgia or systemic lupus erythematosus, depression (controlled or uncontrolled with medications), and narcotic use (none, scheduled, or pro re nata) were retrieved from the electronic medical record (EMR). Patients reported current narcotic use to a perioperative internal medicine specialist whose practice focuses exclusively on medical assessment and optimization prior to and following total joint arthroplasty.

Primary outcomes of pain and opioid consumption during the first 24 hours after surgery were retrieved from the EMR. Patient-reported pain scores recorded by nursing staff on a 10 point scale (ranging from none to severe) every four hours were averaged to derive an overall pain score during the first 24 hours following surgery. Narcotics consumed during the first 24 hours after surgery were recorded and standardized to morphine milligram equivalents using a previously published methodology. [20]

Secondary outcomes related to blood loss also were retrieved from the EMR. Blood loss was evaluated via four metrics: (1) change in preoperative to postoperative day one hemoglobin levels in $\mathrm{g} / \mathrm{dL}$, (2) calculated total blood loss in liters, (3) total hemovac drain output in 
milliliters, and (4) average drain output per hour to account for the variable time drains were in situ. The change in hemoglobin was calculated by subtracting postoperative day one hemoglobin levels from hemoglobin levels obtained at the preoperative medical clearance appointment within thirty days of the index procedure. Total blood loss was calculated using established methodology [21] by multiplying estimated blood volume (EBV) by the change in hemoglobin divided by the average hemoglobin level. EBV was calculated by taking into account the height, weight, and sex of the patient. To determine the estimated blood loss assuming slow or steady blood loss with standard maintenance intravascular fluids, the change in hematocrit, or hemoglobin, over a given time interval has been found to be ideal to determine intraoperative blood loss.[21] However, the formula for intraoperative blood loss has since been modified to better serve the purpose of estimating perioperative blood loss after TKA.[22, 23] Drain output was measured from placement until discontinuation. The use of the last recorded time point of drain output was used to standardize drain output per hour because drain output was not always recorded at the time of discontinuation on the day after surgery. Drain hours were rounded to the nearest fifteen minutes.

All data points used in this study were prospectively collected and entered into the EMR by non-study clinical personnel. Data points were extracted from the EMR without alteration or conversion. Because tourniquet time was collected for the study, data collection was not blinded to study group.

\section{Data Analysis}

Minitab 17 (State College, PA) was used for statistical analysis. Dixon's r22 ratio was used to test continuous variables for statistical outliers. After outliers were identified and removed (total of three data points), Anderson Darling tests were used to evaluate whether 
continuous variables were normally distributed. Student's t-test (t) and Pearson's correlation coefficient (r) were used to compare means of normally distributed continuous variables. Mann Whitney (W) tests and Spearman's rank correlation (rho) were used to compare medians of nonnormally distributed continuous variables. Chi-Square $\left(\mathrm{X}^{2}\right)$ with Fishers $p$ for 2 x 2 tables was used to analyze categorical variables. An alpha level of 0.05 was used to determine statistical significance.

\section{Results}

The average age of patients in the tourniquet (67.7, range 33-91 years) and no tourniquet (67.0, range 47-85 years) groups was not statistically different $(\mathrm{t}=0.58, p=0.561)$. Median BMI in $\mathrm{kg} / \mathrm{m}^{2}$ also did not differ in the two groups $(32.7$ [Q1, Q3 = 28.2, 38.5] vs. 34.2 [Q1, Q3 $=28.7,39.7]$, respectively, $\mathrm{W}=8618.0, p=0.580$ ). There were significantly more females in the tourniquet group $(55.9 \%)$ compared to the no tourniquet group $(44.1 \%)\left(X^{2}=5.945, p=\right.$ 0.019). Consequently, outcome analyses were performed separately for females and males. Sample demographics and covariates are presented separately based on sex and tourniquet use (yes/no) in Table 1. Preoperative depression was 1.6 times higher in female patients for whom a tourniquet was not used compared to those for whom a tourniquet was used $(p=0.047)$. Depression in all patients in both groups was controlled by medication. Age, BMI, procedure time, LOS, and the prevalence of lumbar spine disease, fibromyalgia or systemic lupus erythematosus, and preoperative narcotic use did not statistically differ among females in the two groups ( $p \geq 0.578)$. Among male patients, mean age was higher by 4.6 years $(p=0.046)$ and median procedure time was 7.9 minutes shorter $(p=0.023)$ in the tourniquet group. None of the other demographic characteristics statistically differentiated males with and without tourniquet use $(p \geq 0.146)$. 
Patient-reported pain and opioid consumption are provided separately for females and males and tourniquet use in Table 2. Females who had a tourniquet reported more postoperative pain (median pain score of 2.7 vs. $1.9, p=0.002$ ) and greater opioid consumption (median morphine milligram equivalents of 42.8 vs. $18.8, p<0.001$ ) in the first 24 hours following surgery compared to females without a tourniquet. The presence of depression did not affect median pain scores in the first 24 hours in females with a tourniquet ( 2.6 vs. $2.7, \mathrm{~W}=2017.0, p$ $=0.245$ ) or those without a tourniquet (1.9 vs. $1.8, \mathrm{~W}=1013.5, p=0.923)$. Median morphine milligram equivalents consumed in the first 24 hours by females with a tourniquet ( 40.4 vs. 45.6 , $\mathrm{W}=1954.0, p=0.250)$ and those without a tourniquet $(22.8$ vs. $14.3, \mathrm{~W}=1078.0, p=0.291)$ also did not differ based on the presence of depression (no vs. yes). Time to first opioid did not differ in females with and without a tourniquet $(\mathrm{p}=0.525)$.

There were no differences in self-reported pain $(p=0.192)$, time to first opioid $(p=$ $0.119)$ and amount of opioids consumed $(p=0.203)$ among males with and without a tourniquet (Table 2).

For both females and males, respectively, tourniquet use was associated with significantly lower decreases in mean delta $\mathrm{Hgb}(-2.4$ vs. $-3.0 \mathrm{~g} / \mathrm{dL}, \mathrm{t}=4.15, p<0.001$ and -1.7 vs. $-2.8 \mathrm{~g} / \mathrm{dL}, \mathrm{t}=3.34, p=0.003)$; less mean total blood loss $(-0.9$ vs. -1.1 liters, $\mathrm{t}=3.69, p<$ 0.001 and -0.8 vs. -1.3 liters, $\mathrm{t}=2.69, p=0.013)$; mean total drain output $(220$ vs. $265 \mathrm{ml}, \mathrm{t}=$ $2.08, p=0.040$ and 274 vs. $426 \mathrm{ml}, \mathrm{t}=2.72, p=0.009)$; and mean drain output per hour $(13.7$ vs. $17.3 \mathrm{ml}, \mathrm{t}=2.70, p=0.008$ and 15.9 vs. $23.4 \mathrm{ml}, \mathrm{t}=2.43, p=0.020)$.

\section{Discussion}

Pain after primary TKA can be substantial and has been shown to increase continued opioid use and dependence in previously opioid-naïve [24] and opioid-experienced [25, 26] 
patients. Several studies have observed that tourniquets result in greater pain in the immediate postoperative period, [7-12] with two of those studies [7, 12] showing increased analgesia consumption as a result and one showing no differences in analgesia consumption. [11] None of these studies examined pain and analgesia consumption separately for females and males, and modern perioperative protocols including TXA were not used. More recently, however, evidence of sex differences in knee pain both prior to [27, 28] and after TKA [29, 30] indicating greater pain in females has been reported.

We observed no differences in median pain ( 1.9 vs. 2.3 on a 10 point scale, $p=0.192)$ or opioid consumption (37.1 vs. 39.9 morphine milligram equivalents, $p=0.203$ ) during the first 24 hours after TKA in male patients with and without a tourniquet, respectively. This may be related to existing observations that male patients with symptomatic knee osteoarthritis (OA) have significantly higher thresholds for mechanically-, heat-, and cold-induced pain at the knee than females, [27] and that pain scores adjusted for covariates are significantly lower in males at all levels of OA as measured by the Kellgren and Lawrence System. [28] In addition, consistent with our observations, men reported significantly less pain than women 24 to 36 hours and 24 to 48 hours after TKA, although tourniquet use was not addressed. [29] Unlike our findings, however, the latter study did not find a concomitant sex difference in opioid consumption. In contrast, we found that female patients with and without tourniquets reported higher median pain ( 2.7 vs. $1.9, p=0.002)$ and opioid consumption (42.8 vs. 18.8 morphine milligram equivalents, $p<0.001$ ) in the same period of time. It is noteworthy that median pain scores among females with and without tourniquets were higher than median pain scores in males with and without tourniquets. More importantly, the difference in morphine milligram equivalents 
consumed by female patients with and without tourniquets is equivalent to the difference between $80 \mathrm{mg}$ compared to $35 \mathrm{mg}$ of hydrocodone daily.

With respect to blood loss, consistent with existing literature, tourniquet use resulted in a statistically significant reduction in blood loss for both female $(p \leq 0.040)$ and male $(p \leq 0.020)$ patients, although the total blood savings of approximately 200 milliliters is of unknown clinical significance, especially in non-anemic patients.

Our primary findings suggest that, barring contraindications, switching to tourniquetless TKA for females may reduce opioid analgesia in the postoperative period in this specific demographic without dramatically increasing blood loss or adding additional operative time (Table 1).

Reduced opioid use in the postoperative period is likely to reduce unwanted opioid-related side effects such as constipation, potential for immunosuppression, urinary retention, sedation, and the increased medication load required to reduce these side effects [31] and may lessen the likelihood of chronic opioid dependence. [32] In addition, undesirable consequences associated with tourniquet use including pain, parasthesias, muscle weakness and rare, but devastating vascular injury, would be eliminated.

Limitations of our study include its retrospective design, the unavailability of inpatient pain scores and opioid use beyond the first 24 hours following TKA, and limited data on preoperative narcotic use. We were unable to provide data on inpatient pain and opioid consumption beyond 24 hours because most patients were discharged on postoperative day one resulting in a small number of data points beyond 24 hours. We were able to control for patient narcotic use immediately prior to surgery, but did not have data on long-term narcotic use or dependence. It is a strength of our study that TKAs were performed by a single surgeon at a single academic institution using modern perioperative pain protocols. 
233 America, avoiding tourniquet use during TKA for females may be a relatively risk-free way to 234 decrease opioid consumption during hospitalization. Further study is warranted to elucidate the 235 factors accounting for different outcomes in females and males. 


\section{References}

1. Tai TW, Lin CJ, Jou IM, Chang CW, Lai KA, Yang CY. Tourniquet use in total knee arthroplasty: a meta-analysis. Knee surgery, sports traumatology, arthroscopy : official journal of the ESSKA 19(7): 1121, 2011

2. Alcelik I, Pollock RD, Sukeik M, Bettany-Saltikov J, Armstrong PM, Fismer P. A comparison of outcomes with and without a tourniquet in total knee arthroplasty: a systematic review and meta-analysis of randomized controlled trials. The Journal of arthroplasty 27(3): 331, 2012 3. Yi S, Tan J, Chen C, Chen H, Huang W. The use of pneumatic tourniquet in total knee arthroplasty: a meta-analysis. Archives of orthopaedic and trauma surgery 134(10): 1469, 2014 4. Zhang W, Li N, Chen S, Tan Y, Al-Aidaros M, Chen L. The effects of a tourniquet used in total knee arthroplasty: a meta-analysis. Journal of Orthopaedic Surgery and Research 9: 13, 2014

5. Zhang W, Liu A, Hu D, Tan Y, Al-Aidaros M, Pan Z. Effects of the timing of tourniquet release in cemented total knee arthroplasty: a systematic review and meta-analysis of randomized controlled trials. J Orthop Surg Res 9: 125, 2014

6. Zan PF, Yang Y, Fu D, Yu X, Li GD. Releasing of tourniquet before wound closure or not in total knee arthroplasty: a meta-analysis of randomized controlled trials. The Journal of arthroplasty 30(1): 31, 2015

7. Ejaz A, Laursen AC, Kappel A, Laursen MB, Jakobsen T, Rasmussen S, Nielsen PT. Faster recovery without the use of a tourniquet in total knee arthroplasty. Acta orthopaedica 85(4): 422, 2014 
8. Kumar N, Yadav C, Singh S, Kumar A, Vaithlingam A, Yadav S. Evaluation of pain in bilateral total knee replacement with and without tourniquet; a prospective randomized control trial. Journal of Clinical Orthopaedics and Trauma 6(2): 85, 2015

9. Liu D, Graham D, Gillies K, Gillies RM. Effects of tourniquet use on quadriceps function and pain in total knee arthroplasty. Knee surgery \& related research 26(4): 207, 2014 10. Zhou K, Ling T, Wang H, Zhou Z, Shen B, Yang J, Kang P, Pei F. Influence of tourniquet use in primary total knee arthroplasty with drainage: a prospective randomised controlled trial. $\mathrm{J}$ Orthop Surg Res 12(1): 172, 2017

11. Vandenbussche E, Duranthon L-D, Couturier M, Pidhorz L, Augereau B. The effect of tourniquet use in total knee arthroplasty. International Orthopaedics 26(5): 306, 2002

12. Abdel-Salam A, Eyres KS. Effects of tourniquet during total knee arthroplasty. A prospective randomised study. The Journal of bone and joint surgery British volume 77(2): 250, 1995

13. Office of National Drug Control Policy UEOotP, United States of America. Epidemic: Responding to America's Prescription Drug Abuse Crisis. In. 2011

14. Levy B, Paulozzi L, Mack KA, Jones CM. Trends in Opioid Analgesic-Prescribing Rates by Specialty, U.S., 2007-2012. American journal of preventive medicine 49(3): 409, 2015

15. Volkow ND, McLellan TA, Cotto JH, Karithanom M, Weiss SRB. Characteristics of Opioid Prescriptions in 2009. JAMA : the journal of the American Medical Association 305(13): 1299, 2011

16. American Academy of Orthopaedic Surgeons. Opioid Use, Misuse, and Abuse in Orthopaedic Practice. In. 2015 
17. Hansen CA, Inacio MCS, Pratt NL, Roughead EE, Graves SE. Chronic Use of Opioids Before and After Total Knee Arthroplasty: A Retrospective Cohort Study. The Journal of arthroplasty 32(3): 811, 2017

18. Kim KY, Anoushiravani AA, Chen KK, Roof M, Long WJ, Schwarzkopf R. Preoperative Chronic Opioid Users in Total Knee Arthroplasty-Which Patients Persistently Abuse Opiates Following Surgery? The Journal of arthroplasty 33(1): 107, 2018

19. Politzer CS, Kildow BJ, Goltz DE, Green CL, Bolognesi MP, Seyler TM. Trends in Opioid Utilization Before and After Total Knee Arthroplasty. The Journal of arthroplasty, 2017

20. Bagsby DT, Ireland PH, Meneghini RM. Liposomal bupivacaine versus traditional periarticular injection for pain control after total knee arthroplasty. The Journal of arthroplasty 29(8): 1687, 2014

21. Nadler SB, Hidalgo JH, Bloch T. Prediction of blood volume in normal human adults. Surgery 51(2): 224, 1962

22. Gross JB. Estimating allowable blood loss: corrected for dilution. Anesthesiology 58(3): 277, 1983

23. McConnell J, Dillon J, Kinninmonth A, Sarungi M, Picard F. Blood loss following total knee replacement is reduced when using computer-assisted versus standard methods. Acta orthopaedica Belgica 78(1): 75, 2012

24. Sun EC, Darnall BD, Baker LC, Mackey S. Incidence of and Risk Factors for Chronic Opioid Use Among Opioid-Naive Patients in the Postoperative Period. JAMA internal medicine 176(9): 1286, 2016

25. Zywiel MG, Stroh DA, Lee SY, Bonutti PM, Mont MA. Chronic opioid use prior to total knee arthroplasty. The Journal of bone and joint surgery American volume 93(21): 1988, 2011 
302

303

304

305

306

307

308

309

310

311

312

313

314

315

316

317

318

319

320

321

322

323

324

26. Smith SR, Bido J, Collins JE, Yang H, Katz JN, Losina E. Impact of Preoperative Opioid Use on Total Knee Arthroplasty Outcomes. The Journal of bone and joint surgery American volume 99(10): 803, 2017

27. Bartley EJ, King CD, Sibille KT, Cruz-Almeida Y, Riley JL, 3rd, Glover TL, Goodin BR, Sotolongo AS, Herbert MS, Bulls HW, Staud R, Fessler BJ, Redden DT, Bradley LA, Fillingim RB. Enhanced Pain Sensitivity Among Individuals With Symptomatic Knee Osteoarthritis: Potential Sex Differences in Central Sensitization. Arthritis care \& research 68(4): 472, 2016 28. Glass N, Segal NA, Sluka KA, Torner JC, Nevitt MC, Felson DT, Bradley LA, Neogi T, Lewis CE, Frey-Law LA. Examining sex differences in knee pain: the multicenter osteoarthritis study. Osteoarthritis and cartilage 22(8): 1100, 2014

29. Pope D, El-Othmani MM, Manning BT, Sepula M, Markwell SJ, Saleh KJ. Impact of Age, Gender and Anesthesia Modality on Post-Operative Pain in Total Knee Arthroplasty Patients. The Iowa orthopaedic journal 35: 92, 2015

30. Liu SS, Buvanendran A, Rathmell JP, Sawhney M, Bae JJ, Moric M, Perros S, Pope AJ, Poultsides L, Della Valle CJ, Shin NS, McCartney CJ, Ma Y, Shah M, Wood MJ, Manion SC, Sculco TP. A cross-sectional survey on prevalence and risk factors for persistent postsurgical pain 1 year after total hip and knee replacement. Regional anesthesia and pain medicine 37(4): 415,2012

31. Benyamin R, Trescot AM, Datta S, Buenaventura R, Adlaka R, Sehgal N, Glaser SE, Vallejo R. Opioid complications and side effects. Pain physician 11(2 Suppl): S105, 2008

32. Furlan AD, Hassan S, Famiyeh IM, Wang W, Dhanju J. Long-term opioid use after discharge from inpatient musculoskeletal rehabilitation. Journal of rehabilitation medicine 48(5): 464, 2016 


\section{Acknowledgements}

The project described was supported by the Indiana University Health - Indiana School of Medicine Strategic Research Initiative. 


\begin{tabular}{|c|c|c|c|c|c|c|c|c|}
\hline & \multicolumn{4}{|c|}{ FEMALES } & \multicolumn{4}{|c|}{ MALES } \\
\hline & Tourniquet & \begin{tabular}{|c|} 
No \\
Tourniquet
\end{tabular} & Statistic & $p$ & Tourniquet & $\begin{array}{c}\text { No } \\
\text { Tourniquet }\end{array}$ & Statistic & $p$ \\
\hline $\mathbf{N}$ & 76 & 60 & & & 17 & 31 & & \\
\hline Mean (Range) Age in Years & $66.9(33-91)$ & $67.2(47-85)$ & $\mathrm{t}=0.20$ & 0.844 & $71.3(61-83)$ & $66.7(47-83)$ & $t=2.05$ & 0.046 \\
\hline Mean (Range) BMI (kg/m2) & $33.5(20-47)$ & $33.8(21-48)$ & $\mathrm{t}=0.27$ & 0.788 & $33.5(24-45)$ & $34.4(23-50)$ & $\mathrm{t}=0.44$ & 0.664 \\
\hline $\begin{array}{l}\text { Median (Q1, Q3) Female/Mean } \\
\text { (Range) Male Procedure Time in } \\
\text { Minutes }\end{array}$ & $74.0(69: 80)$ & $74.5(67: 82)$ & $\mathrm{W}=4158.5$ & 0.833 & $77.3(61-96)$ & $85.2(69-106)$ & $\mathrm{t}=2.40$ & 0.023 \\
\hline $\begin{array}{l}\text { Median (Q1, Q3) Limb Ischemia } \\
\text { Time in Minutes }\end{array}$ & $70.0(65: 75)$ & 0.0 & $*$ & $*$ & $70.0(65: 80)$ & 0.0 & $*$ & $*$ \\
\hline $\begin{array}{l}\text { Median (Q1, Q3) Length of Stay } \\
\text { in Days }\end{array}$ & $1(1: 1)$ & $1(1: 1)$ & $\mathrm{W}=4044.0$ & 0.702 & $1(1: 1.5)$ & $1(1: 1)$ & $\mathrm{W}=733.5$ & 0.396 \\
\hline \% with Lumbar Spine Disease & 18.4 & 15.0 & $X^{2}=0.279$ & 0.651 & 41.2 & 22.6 & $\mathrm{X}^{2}=1.838$ & 0.201 \\
\hline $\begin{array}{l}\% \text { with Fibromyalgia or } \\
\text { Systemic Lupus Erythematosus }\end{array}$ & 7.9 & 6.7 & $X^{2}=0.074$ & 1.000 & 0.0 & 0.0 & -- & -- \\
\hline$\%$ with Depression & 27.6 & 45.0 & $\mathrm{X}^{2}=4.429$ & 0.047 & 0.0 & 16.1 & $X^{2}=3.061$ & 0.146 \\
\hline \% with Uncontrolled Depression & 0.0 & $\circ$ & $* *$ & $* *$ & -- & 0.0 & $* *$ & $* *$ \\
\hline \multicolumn{9}{|l|}{$\%$ with Preoperative Narcotic Use } \\
\hline None & 65.8 & 71.7 & $X^{2}=0.647$ & 0.724 & 64.7 & 67.7 & $X^{2}=0.065$ & 0.968 \\
\hline Scheduled & 6.6 & 6.6 & & & 11.8 & 9.7 & & \\
\hline PRN & 27.6 & 21.7 & & & 25.5 & 22.6 & & \\
\hline
\end{tabular}




\begin{tabular}{|c|c|c|c|c|c|c|c|c|}
\hline & \multicolumn{4}{|c|}{ FEMALES } & \multicolumn{4}{|c|}{ MALES } \\
\hline & Tourniquet & $\begin{array}{c}\text { No } \\
\text { Tourniquet }\end{array}$ & Statistic & $p$ & Tourniquet & $\begin{array}{c}\text { No } \\
\text { Tourniquet }\end{array}$ & Statistic & $p$ \\
\hline \multicolumn{9}{|l|}{ PAIN } \\
\hline $\begin{array}{l}\text { Median (Q1, Q3) Pain in First } 24 \\
\text { Hours }\end{array}$ & $\begin{array}{c}2.7 \\
(1.8,3.6) \\
\end{array}$ & $\begin{array}{c}1.9 \\
(1.1,2.7) \\
\end{array}$ & $\mathrm{W}=3408.0$ & 0.002 & $\begin{array}{c}1.9 \\
(0.8,2.7) \\
\end{array}$ & $\begin{array}{c}2.3 \\
(1.7,3.3) \\
\end{array}$ & $\mathrm{W}=820.5$ & 0.192 \\
\hline \multicolumn{9}{|l|}{ OPIOID USE } \\
\hline $\begin{array}{l}\text { Median (Q1, Q3) Time to First } \\
\text { Opioid in Minutes }\end{array}$ & $\begin{array}{c}193 \\
(123,323)\end{array}$ & $\begin{array}{c}183 \\
(106,343)\end{array}$ & $\mathrm{W}=3840.0$ & 0.525 & $\begin{array}{l}260(178 \\
331)\end{array}$ & $\begin{array}{c}172 \\
(108,268)\end{array}$ & $\mathrm{W}=661.5$ & 0.119 \\
\hline $\begin{array}{l}\text { Median (Q1, Q3) Amount of } \\
\text { Opioids in First } 24 \text { Hours in } \\
\text { Morphine Milligram Equivalents }\end{array}$ & $\begin{array}{c}42.8 \\
(28.5,64.8)\end{array}$ & $\begin{array}{c}18.8 \\
(11.4,34.2)\end{array}$ & $\mathrm{W}=2885.5$ & $<0.001$ & $\begin{array}{c}37.1 \\
(24.2,57.0)\end{array}$ & $\begin{array}{c}39.9 \\
(34.2,68.4)\end{array}$ & $\mathrm{W}=819.0$ & 0.203 \\
\hline
\end{tabular}

